\title{
Calling Song Recognition in Female Crickets: Temporal Tuning of Identified Brain Neurons Matches Behavior
}

\author{
Konstantinos Kostarakos and Berthold Hedwig \\ Department of Zoology, University of Cambridge, Cambridge CB2 3EJ, United Kingdom
}

Phonotactic orientation of female crickets is tuned to the temporal pattern of the male calling song. We analyzed the phonotactic selectivity of female crickets to varying temporal features of calling song patterns and compared it with the auditory response properties of the ascending interneuron $\mathrm{AN} 1$ (herein referred to as $\mathrm{TH} 1-\mathrm{AC} 1$ ) and four newly identified local brain neurons. The neurites of all brain neurons formed a ring-like branching pattern in the anterior protocerebrum that overlapped with the axonal arborizations of TH1-AC1. All brain neurons responded phasically to the sound pulses of a species-specific chirp. The spike activity of TH1-AC1 and the local interneuron, B-LI2, copied different auditory patterns regardless of their temporal structure. Two other neurons, B-LI3 and B-LC3, matched the temporal selectivity of the phonotactic responses but also responded to some nonattractive patterns. Neuron B-LC3 linked the bilateral auditory areas in the protocerebrum. One local brain neuron, B-LI4, received inhibitory as well as excitatory synaptic inputs. Inhibition was particularly pronounced for nonattractive pulse patterns, reducing its spike activity. When tested with different temporal patterns, B-LI4 exhibited bandpass response properties; its different auditory response functions significantly matched the tuning of phonotaxis. Temporal selectivity was established already for the second of two sound pulses separated by one species-specific pulse interval. Temporal pattern recognition in the cricket brain occurs within the anterior protocerebrum at the first stage of auditory processing. It is crucially linked to a change in auditory responsiveness during pulse intervals and based on fast interactions of inhibition and excitation.

\section{Introduction}

From cetaceans to insects, animals use acoustic signals with species-specific patterns for intraspecific acoustic communication. Processing and recognition of these signals is crucial to their lifestyle as it is linked to mating success and rivalry behavior (Bradbury and Vehrenkamp, 1998). Elaborate acoustic patterns with variations in sound frequency, amplitude, and duration are used by mammals, birds, and some amphibians (Fay, 1992; Yu and Margoliash, 1996). Communication signals are shaped by sexual selection (Endler and Basolo, 1998; Ryan, 1998). As a result, in some groups of lower vertebrates (e.g., fish and frogs), as well as in different species of insects, these signals evolved based on variation in their temporal structure (e.g., pulse duration, pulse interval, or the combination of pulses within a call pattern) (Bradbury and Vehrenkamp, 1998; Gerhardt and Huber, 2002). Insects tend to use rather simple sequences of sound pulses, in which the feature for species recognition is the temporal pattern of these pulses (Pollack and Hoy, 1979; Pollack, 2000; Gerhardt

\footnotetext{
Received March 9, 2012; revised May 11, 2012; accepted May 17, 2012.

Author contributions: B.H. designed research; K.K. performed research; K.K. analyzed data; K.K. and B.H. wrote the paper.

This work was supported by a Newton International Fellowship from the Royal Society; additional support was given by the Newton Trust Cambridge and the Department of Zoology. Behavioural studies were supported by the Biotechnology and Biological Sciences Research Council; we thank L. Goldrick for excellent technical assistance and H. ter Hofstede and T. Bayley for critically reading the manuscript.

Correspondence should be addressed to Dr. Berthold Hedwig, Department of Zoology, University of Cambridge, Downing Street, Cambridge CB2 3EJ, UK. E-mail: bh202@cam.ac.uk.

DOI:10.1523/JNEUROSCI.1170-12.2012

Copyright $\odot 2012$ the authors $\quad 0270-6474 / 12 / 329601-12 \$ 15.00 / 0$
}

and Huber, 2002). Consequently, on the receiver side, intraspecific communication requires neural networks tuned to the species-specific temporal pattern of the sound signals.

Considerable progress has been made in analyzing the neural mechanisms underlying temporal processing in frogs and fish. Pulse rate selectivity of neurons in the anuran inferior colliculus results from precisely timed interactions between inhibition and pulse rate-dependent excitation (Edwards et al., 2007; Rose et al., 2011). In the fish Pollimyrus, pattern-selective neurons within the torus semicircularis exhibit long-lasting inhibition and postinhibitory rebound properties (Crawford, 1997; Large and Crawford, 2002).

Further to these advances in lower vertebrates, the neural networks of insects provide the advantage to analyze and reveal principles of auditory processing at the level of identified neurons (Wohlers and Huber, 1982; Schildberger, 1984; Brodfuehrer and Hoy, 1990; Zorović and Hedwig, 2011). Phonotactic behavior of female crickets is tuned to the temporal structure of the male calling song. Several concepts have been proposed to explain the neural basis of this selectivity. Hennig (2003) suggested a crosscorrelation analysis between the perceived signal and an innate template based on pulse coincidence. Hoy (1978) speculated that such an internal template could be derived from the same neural network that drives the central pattern generator for singing in males. Data from Bush and Schul (2006) indicate that temporal pattern recognition may be achieved by oscillatory responses of individual neurons. Based on intracellular recordings of auditory brain neurons, Schildberger (1984) suggested that temporal filtering is established by sequential processing in low-pass and 
high-pass filter neurons that finally shape the bandpass response properties of brain neurons that match the temporal tuning of female phonotaxis.

Although these concepts provide hypotheses for pattern recognition, the actual neural mechanisms for temporal selectivity in the cricket brain are still not clearly resolved. Here, we characterize the temporal filtering of auditory brain neurons and compare it with the phonotactic responses of females. We demonstrate that the behavioral selectivity to temporal features is mirrored in the response properties of these brain neurons, and we provide evidence for underlying processing mechanisms.

\section{Materials and Methods}

Last instar female crickets (Gryllus bimaculatus de Geer) were collected from the colony at the Department of Zoology of University of Cambridge (Cambridge, UK) and maintained on a $12 \mathrm{~h}$ light/dark cycle. They were kept isolated from singing males and were used for experiments 1 week after their final moult. All experiments were performed at $21-23^{\circ} \mathrm{C}$.

\section{Behavior}

Females were tethered in natural walking posture on top of a trackball. An L-shaped insect pin $(0.2 \mathrm{~mm}$ diameter $)$ was waxed to the metathoracic tergite and its end clamped into a needle holder. While the females moved during phonotactic walking, an optical sensor (ADNS-2051; 2D optical mouse sensor; Agilent) monitored the rotations of the trackball in the forward-backward and left-right directions. Due to the tethered body position, the sound conditions at the spiracles remained constant during walking (for details, see Hedwig and Poulet, 2004, 2005). In behavioral experiments, each acoustic paradigm was tested three times in at least 10 females; different animals were used for the neurophysiological experiments.

\section{Acoustic stimulation}

The calling song of male G. bimaculatus consists of sound pulses with a duration of 15-23 ms and pulse intervals (time from the end of one pulse to the start of the next) of 16-24 ms. Three to four pulses are grouped into chirps, which are repeated at intervals of $190-250 \mathrm{~ms}$ with an overall chirp rate of 2-3 Hz (Doherty, 1985b; Ferreira and Ferguson, 2002; Verburgt et al., 2010).

We designed three acoustic paradigms to analyze the temporal selectivity of phonotactic behavior and of auditory neurons.

Pulse duration. Using a constant pulse period of $40 \mathrm{~ms}$, four sound pulses were grouped into chirps. Pulse duration (PD) values of 4, 6, 8, 10, $15,20,25,30,35$, and $40 \mathrm{~ms}$ were used. With increasing PD, the pulse interval decreased correspondingly, so a PD of $10 \mathrm{~ms}$ was followed by a pulse interval of $30 \mathrm{~ms}$ and a PD of $40 \mathrm{~ms}$ resulted in a constant tone of $160 \mathrm{~ms}$. This paradigm also comes with a variation of the duty cycle as the pulse period was kept constant at $40 \mathrm{~ms}$ for all chirps. It corresponds to the duty cycle paradigm used by Hennig (2009) and Verburgt et al. (2008).

Pulse interval. Four sound pulses with a constant PD of $20 \mathrm{~ms}$ were grouped into a chirp. The pulse interval (PI) was varied from $0,5,10,15$, $20,25,30,35,40,50,60,80$, to $100 \mathrm{~ms}$. As the sound pulses had a fixed duration of $20 \mathrm{~ms}$, a PI of $0 \mathrm{~ms}$ resulted in a constant tone of $80 \mathrm{~ms}$ and chirps with a PI of $100 \mathrm{~ms}$ had a duration of $380 \mathrm{~ms}$.

Pulse period. Corresponding to the paradigm of Thorson et al. (1982), the pulse period (PP) depends on the variation of two parameters; PD and PI were both increased from 5 to $49 \mathrm{~ms}$ in $4 \mathrm{~ms}$ increments. The resulting $\mathrm{PP}$ of $10,18,26,34,42,50,58,66,74,82,90$, and $98 \mathrm{~ms}$ all had equal PD and PI length (duty cycle of $50 \%$ ). With increasing PP, the number of sound pulses was adjusted to keep the chirp duration close to $240 \mathrm{~ms}$ (Thorson et al., 1982). Accordingly, at a PP of $10 \mathrm{~ms}$, chirps consisted of 25 sound pulses and consisted of 14, 10, 8, 6, 5, 5, 4, 4, 3, 3, and 3 sound pulses at the other PP, respectively. Chirp durations ranged from 230 to $260 \mathrm{~ms}$, and therefore the paradigms did not all emit the same sound energy.

Additionally, in neurophysiological experiments, we used two test paradigms with a consecutive variation of the pulse intervals. In the first paradigm, six sound pulses with a PD of $21 \mathrm{~ms}$ were presented with a PI of $21 \mathrm{~ms}$ followed by a PI of $31,41,71$, and again $21 \mathrm{~ms}$. The sequence had a duration of $305 \mathrm{~ms}$ and was repeated after an interval of $230 \mathrm{~ms}$. In the second paradigm, two sound pulses with a PD of $20 \mathrm{~ms}$ were presented sequentially while their PI was systematically varied from $0,5,10,15,20$, $25,30,35,40,50,60,80$, to $100 \mathrm{~ms}$. Pairs of pulses were separated by intervals of $230 \mathrm{~ms}$.

All acoustic stimuli had a carrier frequency of $4.8 \mathrm{kHz}$ and a sound intensity of $75 \mathrm{~dB}$ SPL relative to $20 \mu \mathrm{Pa}$. The rising and falling ramps for each sound pulse were $2 \mathrm{~ms}$. For behavioral and neurophysiological experiments, acoustic paradigms were presented in a different way. In behavior, chirps of one paradigm were presented for $30 \mathrm{~s}$ from the left-hand and then $30 \mathrm{~s}$ from the right-hand side to test the phonotactic response. The chirp period was constant for all chirps of a paradigm. In neurophysiological experiments, the different chirps of all three paradigms were combined to a sequence and presented once per loop; chirps were separated by a constant chirp interval of $230 \mathrm{~ms}$. The sequenced paradigm was necessary for a comprehensive neural analysis and was appropriate to reveal neural response functions. We analyzed the number of action potentials (APs) generated per chirp, which provided a robust measure for the degree of neural activation and tuning.

Sound stimuli were generated with Cool Edit Pro 2000 software (now Adobe Audition software; Adobe Systems). Signals from the PC audio boards were amplified by a custom-made amplifier and presented by speakers (Sinus Live NEO 13 S; Conrad Electronic), which were placed at an angular position of $45^{\circ}$ to the left and right side of the front of the females at a distance of $30 \mathrm{~cm}$ from the front legs. Sound pressure levels were calibrated at the position of the ears to an accuracy of $1 \mathrm{~dB}$ using a Bruel and Kjaer measuring amplifier (type 2610) and a 1/2 inch free field microphone (type 4191).

\section{Intracellular recordings}

Crickets were placed ventral surface down on a Plasticine block with their legs restrained by metal clamps. The head was slightly tilted backwards and fixed tightly with beeswax into a modified Eppendorf tube attached to a metal rod. The antennae were removed, and the frontal part of the head capsule was opened to expose the ventral side of the brain (for details, see Zorović and Hedwig, 2011). The brain was covered with insect saline [ionic composition (in mmol/L): $140 \mathrm{NaCl}, 10 \mathrm{KCl}, 4 \mathrm{CaCl}_{2}, 4$ $\mathrm{NaHCO}_{3}, 6 \mathrm{NaH}_{2} \mathrm{PO}_{4}$ ]. After exposing the brain, the animals were tethered on a trackball in natural posture. The brain was stabilized between a small metal platform at its dorsal side and a metal ring at its ventral side. The platform served as a reference electrode for intracellular recordings. In some crickets, we used protease (Sigma-Aldrich) to soften the brain perineurium.

A DMZ-Universal micropipette puller (Zeitz Instruments) was used to produce microelectrodes (Harvard Apparatus; $1 \mathrm{~mm}$ OD, $0.58 \mathrm{~mm}$ ID). The microelectrodes were filled with 5\% Lucifer yellow $\mathrm{CH}$ (SigmaAldrich) dissolved in aqua destillata, or with $0.8 \%$ Alexa 568 hydrazid (Invitrogen) dissolved in $0.2 \mathrm{~m}$ lithium chloride ( $\mathrm{LiCl})$. The shaft of the capillaries was backfilled with $0.5 \mathrm{M} \mathrm{LiCl}$ providing electrodes of $100-140$ or $70-100 \mathrm{M} \Omega$, respectively.

For intracellular recordings, microelectrodes were positioned with a Leitz micromanipulator (model M; Leica Microsystems). Electrode depth was controlled with a Mitutoyo absolute digimatic indicator (ID-C125MB; Mitutoyo Corporation). Auditory brain neurons were encountered at a depth of $20-300 \mu \mathrm{m}$ in the lateral and medial anterior protocerebrum where the axonal projections of the ascending neuron TH1-AC1 terminate. Test pulses of $50 \mathrm{~ms}$ duration and carrier frequencies of $4.8,10$, and $20 \mathrm{kHz}$ were used to evoke field potentials and activate auditory neurons. Intracellular recordings lasted between $30 \mathrm{~s}$ and 20 min and were amplified by a SEC-05LX amplifier (npi electronic) operating in bridge mode.

Fluorescent dyes were iontophoretically injected into the neurons for $5-20 \mathrm{~min}$ by hyperpolarizing current injection $(0.5-5 \mathrm{nA})$. For histological processing, the brain was dissected and fixed in $4 \%$ paraformaldehyde, dehydrated in a series of ethanol at 70,90,95, and 100\%, and finally cleared in methyl-salicylate. A Zeiss Axiophot epifluorescence microscope (Carl Zeiss) was used to visualize the morphology of the neurons 

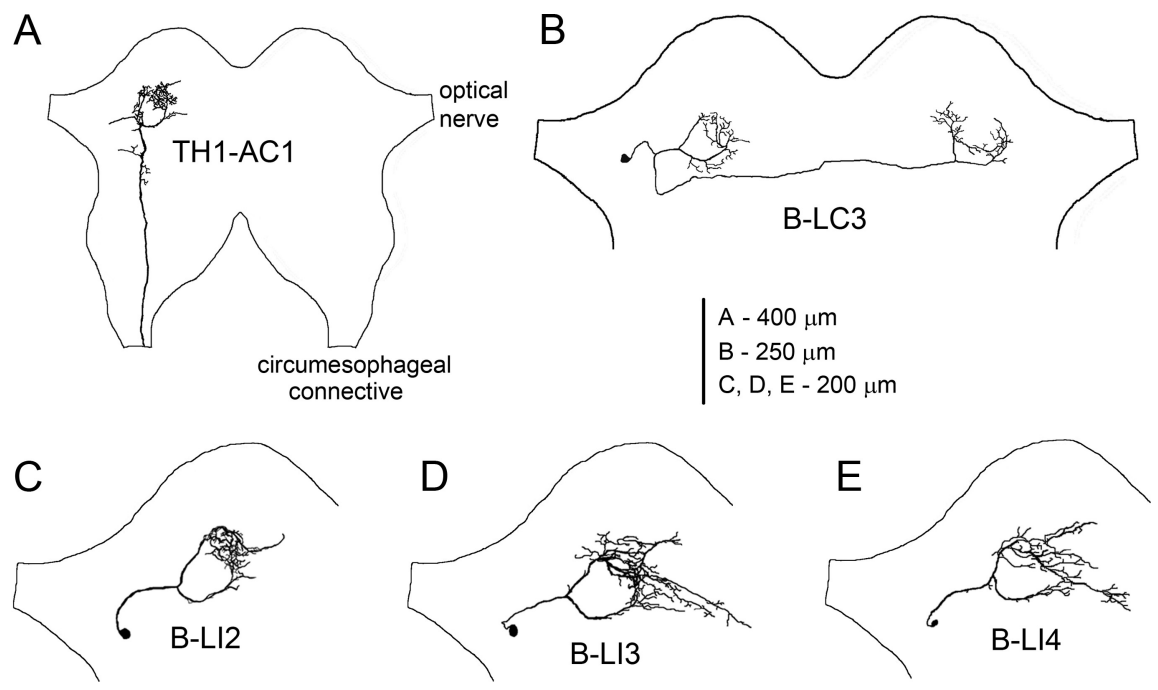

Figure 1. Structure and location of auditory neurons in the cricket brain. $A$, Axonal projections of ascending neuron TH1-AC1 in the anterior protocerebrum. $B$, Morphology of $B-L C 3$ with a cell body next to the optic nerve; dendritic arborizations overlap the ring-like arborization patterns of the ipsilateral TH1-AC1 and axonal projections overlap the contralateral one. $C-E$, Morphology of B-LI2, B-LL3, and B-LI4 with a lateral cell body.

using Zeiss filter sets $63 \mathrm{HE}$ for Alexa and $73 \mathrm{HE}$ for Lucifer yellowlabeled neurons. Images were taken with a digital SLR camera (Canon EOS 350D; Canon) attached to the microscope. Neurons were reconstructed manually from image stacks using ImageG (National Institutes of Health, Bethesda, MD) and Photoshop CS4 (Adobe Systems) software. Neurons were identified according to their morphology and response patterns.

\section{Data recordings and analysis}

All recording channels were digitized at $17.8 \mathrm{kHz}$ and 16 bit amplitude resolution with $0.153 \mathrm{mV}$ per increment using a CED 1401 plus data acquisition interface. Data were recorded to the hard disc of a PC using Spike 2 software (Cambridge Electronic Design). Neural recordings were also displayed on an oscilloscope (5103N; Tektronix) and monitored using headphones. Behavioral data and neural recordings were analyzed off-line using customized Neurolab software (Hedwig and Knepper, 1992; Knepper and Hedwig, 1997). Phonotactic steering was calculated from the lateral deviation toward the left or right sound source (for details, see Hedwig and Poulet, 2004, 2005). To compare the behavioral and neurophysiological responses, we calculated for identical acoustic paradigms the relative values of the phonotactic response and also of the spike activity. For each paradigm, the maximal response was set to $100 \%$, and all other responses were expressed relative to this. The relative responses were then averaged over all tested animals. Neural responses were also analyzed using peristimulus time (PST) histograms and averages of instantaneous spike frequency. To further relate the tuning curves of neural responses to the tuning of the phonotactic behavior, we calculated the correlation coefficient $(r)$ between both sets of data. For plotting pulse duration/pulse interval response profiles (see Fig. 6), we used SigmaPlot (Systat Software).

\section{Terminology}

Brain neurons were labeled with a sequence of letters and numbers (Hedwig, 1986; Zorović and Hedwig, 2011). The first letter indicates the ganglion that contains the cell body (B for brain), the second letter describes axonal projections to other ganglia (e.g., L for local, D for descending), and the third letter indicates if the axon runs ipsilateral or contralateral to the cell body. The ascending interneuron, AN1 (Wohlers and Huber, 1982; Schildberger and Hoerner, 1988), is described here as TH1-AC1.

In the following text, we will briefly refer to, for example, a pulse interval of $20 \mathrm{~ms}$ or a pulse period of $34 \mathrm{~ms}$ as PI-20 ms and PP-34 ms, respectively.

\section{Results \\ Structure of identified auditory brain neurons}

In addition to the ascending interneuron TH1-AC1, we identified four local auditory brain neurons in the protocerebrum (Fig. 1). The cell body of TH1-AC1 was located in the prothoracic ganglion from where the axon ascended to the brain and projected to the ventral protocerebrum lateral to the $\alpha$-lobe (Wohlers and Huber, 1982). Here, the axon formed a ring-like projection around a so-far-unidentified neuropil structure; one prominent axonal branch projected medially in an anterior loop, while the other followed a posterior path. The branches met slightly lateral to the ocellar nerve forming dense arborizations (Fig. 1A). This ring-like branching pattern was a common feature of the local auditory brain neurons identified here, overlapping with the axonal projection pattern of TH1-AC1.

All four local interneurons had a cell body in the lateral protocerebrum, next to the optical nerve with a curved primary neurite projecting medially toward the auditory neuropil. The primary neurite then split into two main branches looping around a central structure as described above. Medially, where both neurites met, the neurons formed characteristic but similar dense arborizations, which may be regarded as an auditory neuropil in the anterior protocerebrum. The arborizations of interneuron B-LI2 were rather confined and exhibited no clear separation of axonal or dendritic neurites (Fig. 1C). In contrast, neurons B-LI3 and B-LI4 had a slightly wider arborization pattern and additionally had projections to the midline of the brain with a "beaded" axonal appearance (Fig. $1 D, E$ ). Due to their high structural similarity, these neurons could not be distinguished unequivocally on structural criteria alone. Dendrites of B-LC3 also overlaid the ring-like arborization pattern. Different from previous local interneurons, B-LC3 had an axon projecting to the contralateral auditory neuropil, where it again followed the ring-like projection pattern (Fig. $1 B$ ).

\section{Temporal selectivity of phonotaxis and of the ascending interneuron TH1-AC1}

Phonotactic behavior showed a clear temporal selectivity at different PD, PI, and PP as it revealed a bandpass tuning with maximal responses to specific temporal patterns. The maximal responses occurred at PD-15 ms, PI-15 ms, and PP-34 and $42 \mathrm{~ms}$ (Fig. 2). We collected neurophysiological data from different females than the ones used in behavioral tests.

We first compared the behavioral phonotactic responses with the spike activity of the ascending interneuron TH1-AC1, which is the only ascending interneuron forwarding calling-songspecific information from the prothoracic auditory neuropil toward the brain (Wohlers and Huber, 1982; Schildberger, 1984). The activity of this neuron has been described but never comprehensively compared against phonotactic tuning to different temporal paradigms. Spike activity of interneuron TH1-AC1 was recorded from its main axon in the protocerebrum while the different sound paradigms were presented. TH1-AC1 reliably responded to the sound pulses of all test patterns. 
Response to PD

With increasing PD and simultaneously decreasing PI, the neuron generated an increasing number of spikes (Fig. 2A, top). It responded to each sound pulse at PD-8 ms with a burst of spikes, and a total of 13.7 AP/chirp ( $\mathrm{SD} \pm 1.4)$. At PD-20 ms, the response was $25.7 \pm 3.2 \mathrm{AP} /$ chirp, and at $\mathrm{PD}-40 \mathrm{~ms}$, the response to the nowcontinuous pulse reached $37.2 \pm 5.1 \mathrm{AP} /$ chirp on average. At PD-40 ms, the spike frequency decreased continuously from 323 to $193 \mathrm{~Hz}$ from the beginning to the end of the response. Correspondingly, the $\mathrm{PD}$-response function of the neuron increased over the range of PD tested by $74.6 \%$ (28.0 AP), increasing by $38.8 \%$ (14.5 AP) from PD-10 to $25 \mathrm{~ms}$, but only by $18.9 \%$ (7.1 AP) from PD-25 to $40 \mathrm{~ms}$ (Fig. 2A, bottom).

In contrast, the $\mathrm{PD}$-response function of phonotaxis had the shape of a broadly tuned optimum curve exhibiting a maximal response at PD-15 ms (Fig. 2A, bottom). The phonotactic response increased by $70.4 \%$ from PD-4 to $15 \mathrm{~ms}$ and decreased by $17.4 \%$ from PD-15 to $25 \mathrm{~ms}$ and further by $50.6 \%$ to $40 \mathrm{~ms}$. It reached values $>50 \%$ in the PD range of $8-35 \mathrm{~ms}$. Comparing the PDresponse function of TH1-AC1 with the behavioral tuning revealed that the responses did not match. This was reflected by a correlation coefficient of $r^{2}=0.00(p>0.05)$.

\section{Response to PI}

The response of TH1-AC1 only slightly increased when PI was increased from 0 to $100 \mathrm{~ms}$, while PD was kept constant at $20 \mathrm{~ms}$ (Fig. $2 \mathrm{~B}$ ). With PI-5 ms chirps elicited a continuous burst of spikes with $23.2 \pm 2.8 \mathrm{AP}$, at $\mathrm{PI}-15 \mathrm{~ms}$ the response was $23.8 \pm 3.0$ $\mathrm{AP}$ and it slightly increased to $26.7 \pm 2.7 \mathrm{AP}$ at PI-60 ms (Fig. $2 \mathrm{~B}$, top). Over the range of PI tested, the response function of TH1$\mathrm{AC} 1$ revealed a gradual increase by $25.2 \%$ (7.5 AP).

In comparison, the phonotaxis PI-response function (Fig. $2 B$, bottom) revealed a narrow optimum curve with a maximum characterized by the scores at PI-15 ms (95.2\%) and PI-20 ms (93.8\%). The phonotactic response steeply increased by $74.5 \%$ from PI-5 to $15 \mathrm{~ms}$ and decreased by $42.5 \%$ from PI-20 to $30 \mathrm{~ms}$, dropping further at higher PI. The PI-response function reached values $>50 \%$ within the range of $10-30 \mathrm{~ms}$. The correlation coefficient between both data sets was $r^{2}=-0.18(p>0.05)$ and demonstrated that the response functions did not match.

\section{Response to PP}

When we varied PP between 10 and $98 \mathrm{~ms}$, the overall response of TH1-AC1 revealed only a slight variation. At PP-18 ms, the neuron generated $32.7 \pm 3.9 \mathrm{AP} / \mathrm{chirp}$, at $34 \mathrm{~ms}$ it responded with $36.8 \pm 4.1 \mathrm{AP} / \mathrm{chirp}$, and at PP-90 ms its spike activity was $35.0 \pm$ 4.7 AP/chirp (Fig. 2C). The PP-response function of TH1-AC1 revealed an overall high level of activity as the relative response was $>80 \%$ at all PP presented. The spike activity fluctuated between the maximum response at PP-74 $\mathrm{ms}$ (38.4 $\pm 5.0 \mathrm{AP} / \mathrm{chirp})$ and the minimum response at PP-82 $\mathrm{ms}(32.1 \pm 4.0 \mathrm{AP} /$ chirp $)$. These fluctuations are not due to variability in the neural response, but mirror the slight differences in sound energy presented for different pulse periods, as the number of sound pulses per chirp was adapted to match the chirp duration (see Materials and Methods). Very similar fluctuations were also described for a local thoracic auditory interneuron by Nabatiyan et al. (2003).

In response to the PP paradigm, female phonotaxis exhibited a bandpass like tuning as previously described (Thorson et al., 1982; Doherty, 1985a; Hedwig, 2006) and had a maximal response of $93.9 \%$ at PP-34 and $42 \mathrm{~ms}$. From the maximum, the response function decreased with a similar slope toward lower and higher PP. When PP increased from 18 to $34 \mathrm{~ms}$, phonotaxis increased by $73.8 \%$ and it decreased in a similar way by $71.0 \%$ when PP changed from 42 to $58 \mathrm{~ms}$. The relative phonotactic response was $>50 \%$ within a $\mathrm{PP}$ range of $26-50 \mathrm{~ms}$ and $>80 \%$ only at the response maxima of 34 and $42 \mathrm{~ms}$. In comparison, the relative activity of $\mathrm{TH} 1-\mathrm{AC} 1$ was $>80 \%$ for all $\mathrm{PP}$ and did not reveal any tuning; the correlation coefficient with behavior was $r^{2}=0.00(p>0.05)$

\section{Tuning of local brain neurons}

To identify brain neurons with response properties that match the selectivity of phonotaxis, we systematically probed the anterior protocerebrum and analyzed the spike activity of auditory neurons. In the following, we present the auditory response properties of four local brain neurons, B-LI2, B-LC3, B-LI3, and B-LI4, and compare their temporal tuning with that of phonotaxis. A statistical treatment of the neural data is given in Table 1.

\section{Impact of $P D$}

When tested with different PD, female crickets exhibited a broadly tuned phonotactic response with a maximum at PD-15 ms (Fig. 2A). When stimulated with the PD paradigm, interneuron B-LI2 was rhythmically depolarized by the pattern of sound pulses and generated two to three spikes per pulse (Fig. 3A). For all PD patterns tested, the response at the beginning of a chirp was slightly stronger. With increasing pulse duration, the response of the neuron increased from $8.1 \pm 0.9 \mathrm{AP} /$ chirp at PD-8 ms to 
Table 1. Statistical treatment of neural data

\begin{tabular}{|c|c|c|c|c|c|c|}
\hline \multirow[b]{2}{*}{ Neurons } & \multicolumn{2}{|l|}{$P D$} & \multicolumn{2}{|l|}{$\mathrm{PI}$} & \multicolumn{2}{|l|}{ PP } \\
\hline & Below max & Above max & Below max & Above max & Below max & Above max \\
\hline B-LC3 & $15-20 \mathrm{~ms}$ & $\mathbf{2 0 - 3 5 \mathrm { ms }}$ & $5-15 \mathrm{~ms}$ & $15-25 \mathrm{~ms}$ & $26-34 \mathrm{~ms}$ & $34-42 \mathrm{~ms}$ \\
\hline$N=5$ & $p=0.005$ & $p=0.018$ & $p<0.001$ & $p=0.018$ & $p=0.067$ & $p=0.063^{*}$ \\
\hline B-LI3 & $10-20 \mathrm{~ms}$ & $20-40 \mathrm{~ms}$ & $5-15 \mathrm{~ms}$ & $15-30 \mathrm{~ms}$ & $26-34 \mathrm{~ms}$ & $34-42 \mathrm{~ms}$ \\
\hline$N=5$ & $p=0.008$ & $p=0.100$ & $p=0.001$ & $p=0.01$ & $p=0.008$ & $p=0.013$ \\
\hline B-LI4 & $10-15 \mathrm{~ms}$ & $20-30 \mathrm{~ms}$ & $5-15 \mathrm{~ms}$ & $20-25 \mathrm{~ms}$ & $26-34 \mathrm{~ms}$ & $34-58 \mathrm{~ms}$ \\
\hline$N=3$ & $p=0.052$ & $p=0.042^{* *}$ & $p=0.022^{* *}$ & $p=0.038$ & $p=0.061$ & $p=0.003$ \\
\hline
\end{tabular}

Statistical treatment for significant differences by a paired $t$ test. Data with asterisk were obtained by a Wilcoxon signed rank test for samples that were not normally distributed. The smallest significant differences of relative values below and above the maximal responses (indicated in bold) are shown for each paradigm. Due to the small sample sizes, we considered differences as significant at values of $p \leq 0.1$ in order to avoid errors that can occur at small sample sizes due to large SDs. Data with two asterisks showed no significant differences referred to the maximal response as data were not normally distributed or had a high SD. For these data, we show the smallest significant differences below and above the nearest value to the maximum.

\section{Pulse Duration}

A

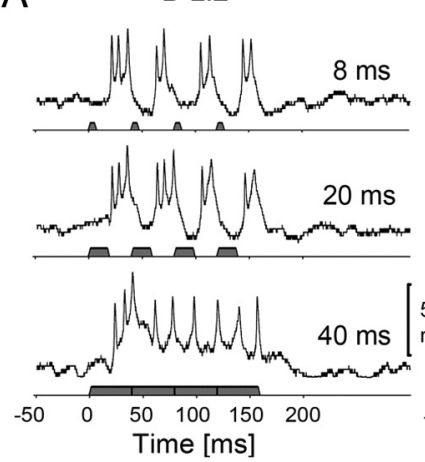

B B-LC3

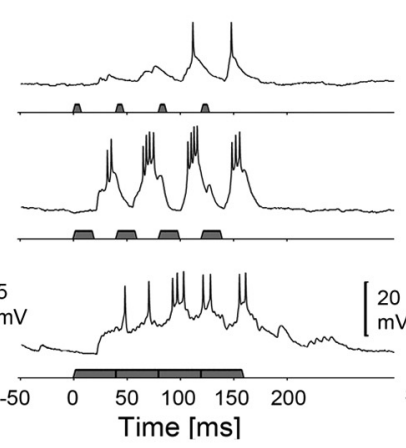

C B-LI3
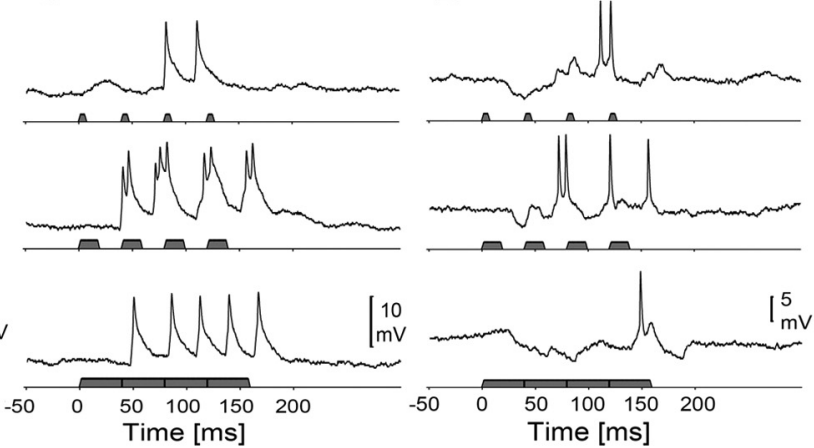

Figure 3. Activity of B-LI2, B-LC3, B-LI3, and B-LI4 and phonotactic tuning of females to different PD. Top, Response of neurons at PD-8, 20, and 40 ms. Bottom, Phonotactic (gray) and neural (black) PD-response functions. $A$, Activity and response function of B-LI2. Neural data are based on $N=2$ females and $n=13$ test sequences, 2 stainings. $B$, Activity and response function of $B$ - $L$ C 3 . Neural data are based on $N=5$ females and $n=23$ test sequences, 4 stainings. C, Activity and response function of B-LI3. Neural data are based on $N=5$ females and $n=18$ test sequences, 4 stainings. D, Activity and response function of B-LI4. Neural data are based on $N=3$ females and $n=10$ test sequences, 2 stainings. The left scale indicates relative neural and phonotactic activity, and the right scale shows the absolute values of spike activity. Error bars show SD of relative values.

$10.9 \pm 4.4 \mathrm{AP} /$ chirp at PD-20 ms and $12.9 \pm 4.9 \mathrm{AP} /$ chirp at $\mathrm{PD}-35 \mathrm{~ms}$; the response at PD-40 ms then slightly fell to $12.1 \pm$ 5.8 AP/chirp (Fig. 3A, top). Correspondingly, the PD-response function of B-LI2 increased by $55.6 \%$ (7.3 AP) from PD-4 ms to $\mathrm{PD}-35 \mathrm{~ms}$, and then decreased by $8.5 \%$ (0.8 AP) from 35 to $40 \mathrm{~ms}$. The response function of B-LI2 revealed no optimum. It did not match with the phonotactic response function (Fig. 3A, bottom) and the correlation coefficient was only $r^{2}=0.10(p>0.05)$. The PD-dependent activity of B-LI2, however, was similar to the response of TH1-AC1 (Fig. 2) and neural PD-response functions of these two neurons exhibited a highly significant correlation of $r^{2}=0.91(p<0.001)$.

The activity of the contralaterally projecting neuron B-LC3 was more complex (Fig. $3 B$ ). At PD-8 ms, the neuron responded with subthreshold EPSPs of $4-8 \mathrm{mV}$ to the first two sound pulses of the chirps; the following pulses elicited suprathreshold EPSPs and triggered spikes. On average, B-LC3 generated $3.6 \pm 1.7$
AP/chirp at PD-8 ms, at PD-20 ms it elicited EPSPs of $15 \mathrm{mV}$ amplitude and $12.0 \pm 1.1 \mathrm{AP} /$ chirp, and at $\mathrm{PD}-40 \mathrm{~ms}$ the neuron showed a continuous depolarization of $10 \mathrm{mV}$ generating $8.8 \pm$ $1.8 \mathrm{AP} /$ chirp. At PD-20 ms, B-LC3 always responded more strongly to the second pulse of a chirp ( $3.4 \pm 0.3 \mathrm{AP} /$ sound pulse $)$ than to the first $(1.8 \pm 0.5 \mathrm{AP} /$ sound pulse $)$. This was different from the previously described responses of TH1-AC1 and B-LI2. The PD-response function of B-LC3 revealed a maximum at PD-20 ms. From PD-4 to $15 \mathrm{~ms}$, spike activity increased by $84.0 \%$ (10.3 AP) but decreased by only $25.6 \%$ (3.1 AP) from PD-20 to 40 $\mathrm{ms}$. The PD-response functions of the behavior and B-LC3 increased in parallel up to PD-15 ms and exhibited a similar maximum at 15-20 ms. However, when PD increased from 20 to 40 $\mathrm{ms}$, phonotaxis decreased more $(60.0 \%)$ than the neural response $(25.6 \%)$ and both response functions matched only weakly with $r^{2}=0.40(p=0.05)$. Although sound was continuous for $160 \mathrm{~ms}$ at $\mathrm{PD}-40 \mathrm{~ms}$, in $60 \%$ of the recorded $\mathrm{B}-\mathrm{LC} 3$, the response tran- 


\section{Pulse Interval}

A

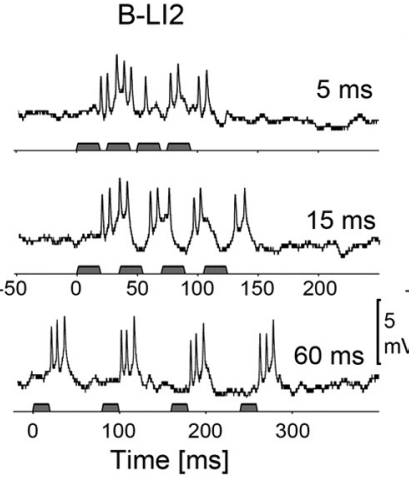

B
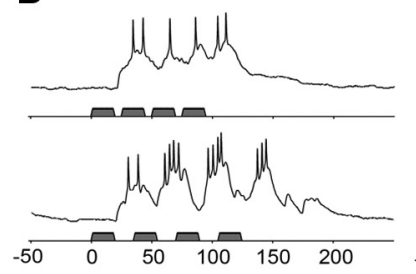
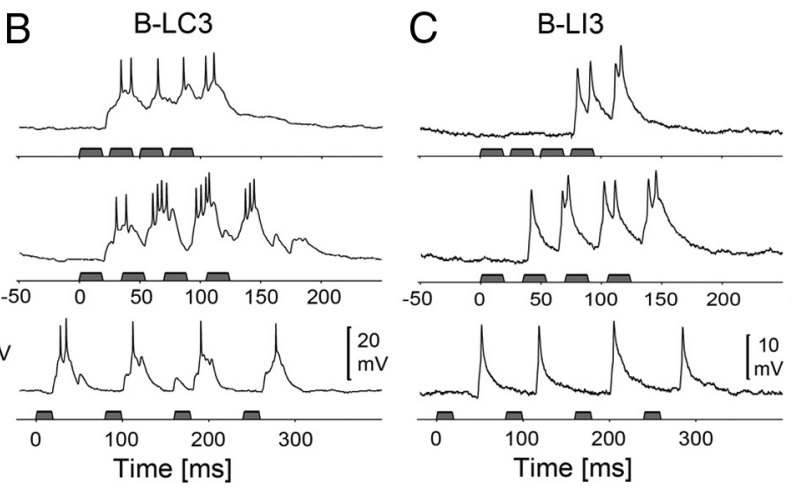

D B-LI4
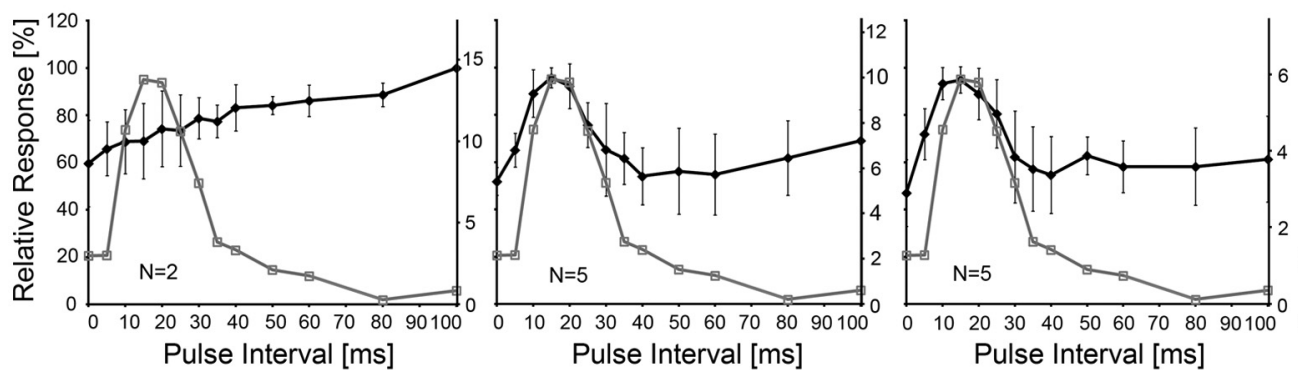

Pulse Interval [ms]
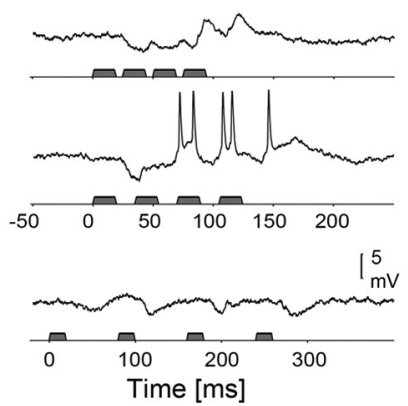

Figure 4. Activity of B-LI2, B-LC3, B-LI3, and B-LI4 and phonotactic tuning of females to different PI. Top, Response of neurons at PI-5, 15, and $60 \mathrm{~ms}$. Bottom, Phonotactic (gray) and neural (black) PD-response functions. $A$, Activity and response function of B-LI2. Neural data are based on $N=2$ females and $n=13$ test sequences, 2 stainings. $B$, Activity and response function of B-LC3. Neural data are based on $N=5$ females and $n=23$ test sequences, 4 stainings. C, Activity and response function of B-LI3. Neural data are based on $N=5$ females and $n=18$ test sequences, 4 stainings. $D$, Activity and response function of B-LI4. Neural data are based on $N=3$ females and $n=10$ test sequences, 2 stainings. The left scale indicates relative neural and phonotactic activity, and the right scale shows the absolute values of spike activity. Error bars show SD of relative values.

siently appeared to be a sequence of rhythmically modulated EPSPs generating bursts of two to three spikes with a mean cycle period of $29 \mathrm{~ms}$.

The response properties of the local interneuron B-LI3 were similar to the response of interneuron B-LC3. At PD-8 ms, B-LI3 (Fig. 3C) generated $2.4 \pm 1.9 \mathrm{AP} /$ chirp; its activity increased to $6.7 \pm 1.1 \mathrm{AP} /$ chirp at PD-20 ms, responding with $10 \mathrm{mV}$ EPSPs and spikes, and at PD-40 ms the activity of the neuron decreased to $4.9 \pm 1.5 \mathrm{AP} /$ chirp. When tested with $\mathrm{PD}-20 \mathrm{~ms}$ pulses, the response to the second pulse of a chirp was higher $(2.1 \pm 0.3$ $\mathrm{AP} /$ sound pulse) than the response to the first pulse $(1 \pm 0.4$ $\mathrm{AP} /$ sound pulse). The $\mathrm{PD}$-response function revealed the strongest response at PD-20 ms. Overall activity increased by $81.1 \%$ (5.7 AP), with PD increasing from 4 to $15 \mathrm{~ms}$ but only gradually decreased by $25.3 \%$ ( $1.8 \mathrm{AP}$ ) when PD further increased from 20 to $40 \mathrm{~ms}$. The PD-response function of B-LI3 matched with the phonotactic behavior slightly better than B-LC3 and gave a correlation coefficient of $r^{2}=0.46(p<0.05)$.

Different from the neural activity described so far, the response of B-LI4 always started with an inhibition (Fig. 3D). At PD- $8 \mathrm{~ms}$, the first sound pulse triggered an IPSP of $4 \mathrm{mV}$ amplitude and $45 \mathrm{~ms}$ duration, which was followed by EPSPs and a mean of $1.9 \pm 0.6 \mathrm{AP} /$ chirp elicited by the consecutive sound pulses. At PD-20 ms, the chirp triggered an initial inhibition that lasted for $15 \mathrm{~ms}$, which was followed by a mixture of IPSPs, EPSPs, and spikes leading to a mean response of $4.2 \pm 1.2 \mathrm{AP} /$ chirp. Chirps with PD-40 ms evoked an initial inhibition of 120 ms duration, varying between -2 and $-3.5 \mathrm{mV}$ and an excitatory response of $0.8 \pm 1.4 \mathrm{AP} /$ chirp generated toward the end of the stimulus. The PD-response function of B-LI4 had the shape of an optimum curve with the maximum spike activity at PD-15 ms (4.7 $\pm 0.2 \mathrm{AP} /$ chirp). With PD increasing from 4 to $15 \mathrm{~ms}$, spike activity increased steeply by $93.5 \%$ (4.6 AP) and different from interneurons B-LC3 and BLI3 then also strongly decreased by 84.8\% (4.1 AP) when PD increased from 15 to $35 \mathrm{~ms}$. Thus, the overall shape of the PD-response function of the neuron significantly matched the phonotactic PD-response giving a correlation coefficient of $r^{2}=0.90(p<0.001)$.

\section{Impact of PI}

When tested with different PI, the phonotactic response of the females revealed a narrowly tuned optimum curve with a maximal response at PI-15 ms. We compared this with the spike pattern of the auditory brain neurons (Fig. 4, top) and their PI-response functions (Fig. 4, bottom).

In neuron B-LI2, spike activity increased with increasing PI. At PI-5 ms, it responded with a continuous burst of $9.5 \pm 2.5$ $\mathrm{AP} /$ chirp; at PI-15 ms, the response was rhythmically coupled to the sound pulses and $10 \pm 3.2 \mathrm{AP} /$ chirp, and at PI-60 ms the activity of the neuron increased to $12.4 \pm 2.0 \mathrm{AP} /$ chirp (Fig. $4 A$ ). The PI-response function revealed a linear increase of $40.4 \%$ (5.7 AP) over the range of $0-100 \mathrm{~ms}$. Correspondingly, the PIresponse of B-LI2 did not match the tuned phonotactic response function as the correlation coefficient was $r^{2}=-0.25(p>0.05)$. The response function was, however, very similar to the PIresponse function of $\mathrm{TH} 1-\mathrm{AC} 1$, as indicated by a correlation coefficient of $r^{2}=0.96$ with $p<0.001$.

In interneuron B-LC3, chirps with PI-5 ms elicited EPSPs of 10-13 mV amplitude, which were modulated by the pulse pattern and generated $6.9 \pm 1.5 \mathrm{AP} /$ chirp (Fig. $4 B$ ). At PI-15 ms, B-LC3 responded with EPSPs of $15 \mathrm{mV}$ amplitude and bursts of spikes to each pulse, generating $10.1 \pm 1.6 \mathrm{AP} / \mathrm{chirp}$. Again, the response of the neuron to the second pulse was stronger than to the first pulse (compare with Figs. 4 B, 3 B). At PI-60 ms, EPSPs 

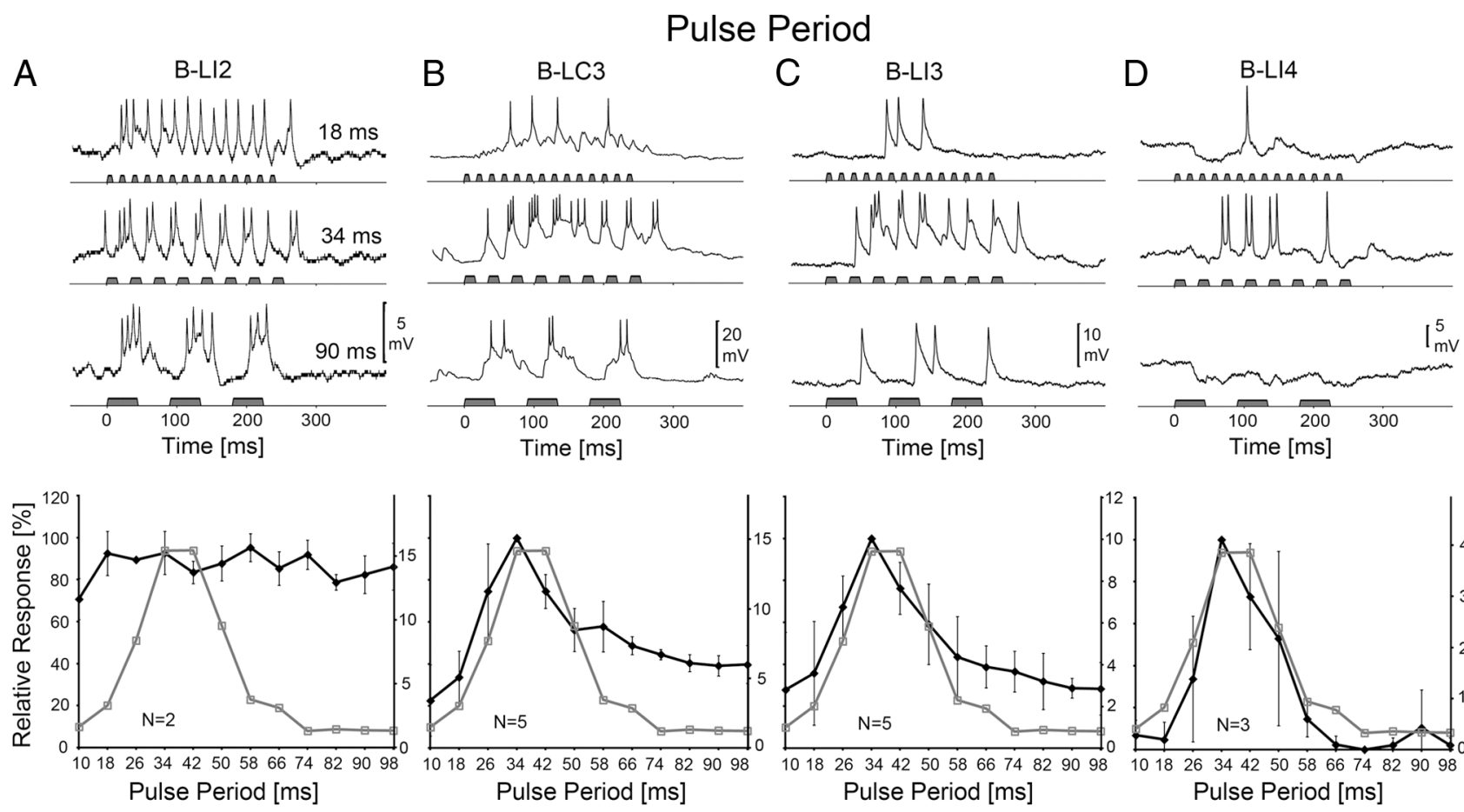

Figure 5. Activity of B-LL2, B-LC3, B-LI3, and B-LI4 and phonotactic tuning of females to different PP. Top, Response of neurons at PP-18, 34, and $90 \mathrm{~ms}$. Bottom, Phonotactic (gray) and neural (black) PD-response functions. $A$, Activity and response function of B-LI2. Neural data are based on $N=2$ females and $n=13$ test sequences, 2 stainings. $B$, Activity and response function of B-LC3. Neural data are based on $N=5$ females and $n=23$ test sequences, 4 stainings. C, Activity and response function of B-LI3. Neural data are based on $N=5$ females and $n=18$ test sequences, 4 stainings. $\boldsymbol{D}$, Activity and response function of B-LI4. Neural data are based on $N=3$ females and $n=10$ test sequences, 2 stainings. The left scale indicates relative neural and phonotactic activity, and the right scale shows the absolute values of spike activity. Error bars show SD of relative values.

were $\sim 10 \mathrm{mV}$ but generated only 1-2 APs per pulse and the spike activity of the neuron decreased to $5.7 \pm 1.8 \mathrm{AP} /$ chirp. The PIresponse function of B-LC3 revealed an optimum curve with maximum activity at PI-15 ms. With PI increasing from 5 to 15 ms, spike activity increased by $30.7 \%$ (3.2 AP). From $20-30 \mathrm{~ms}$, the activity of the neuron decreased by $26.9 \%$ (2.9 AP) and then stayed at a relatively high level of $55-60 \%$ at longer PI. As the tuning of phonotaxis and the PI-response function of the neuron had a similar maximum and shape, there was a highly significant relationship between the two with a correlation coefficient of $r^{2}=0.77(p<0.001)$.

B-LI3 generated 4.5 \pm 0.9 AP to chirps with PI-5 ms (Fig. 4C) but did not respond to the first pulses of a chirp. At PI-15 ms, the response increased to $5.9 \pm 0.9 \mathrm{AP} /$ chirp with the response to the first pulse of the chirp being weaker than the following ones, as with the PD paradigm (Fig. 3C). Activity then decreased to $3.6 \pm$ $0.9 \mathrm{AP} /$ chirp at PI-60 ms. The PI-response function of B-LI3 showed a maximum spike activity at PI- $15 \mathrm{~ms}$. With PI increasing from 5 to $15 \mathrm{~ms}$, activity increased by $23 \%$ (1.4 AP), and between 20 and $30 \mathrm{~ms}$, it decreased by $26.6 \%$ (1.7 AP) and stayed at $\sim 60 \%$ at longer PI. Similar to B-LC3, the PI-response function of B-LI3 matched the phonotactic response with $r^{2}=0.75(p<0.001)$ and high significance.

The best agreement between neural and phonotactic tuning was obtained for interneuron B-LI4 (Fig. $4 D$ ). At PI-5 ms, the response of the neuron started with IPSPs of $-3.6 \mathrm{mV}$ and only toward the end of the chirp did it generate EPSPs with $0.2 \pm 0.3$ $\mathrm{AP} /$ chirp. At PI-15 ms, the response of the neuron began with an inhibition of $-4 \mathrm{mV}$ amplitude and $18 \mathrm{~ms}$ duration, but the consecutive spike activity increased to $2.9 \pm 1.8 \mathrm{AP} /$ chirp. When PI was increased to $60 \mathrm{~ms}$, sound pulses generally elicited IPSPs of $-2.5 \mathrm{mV}$ amplitude and $19-35 \mathrm{~ms}$ duration followed by a weak spike activity of $0.2 \pm 0.4 \mathrm{AP} /$ chirp. The PI-response function of $\mathrm{B}-\mathrm{LI} 4$ revealed a narrowly tuned optimum curve with a maximal response of $3 \pm 1.5 \mathrm{AP} /$ chirp at PI-20 ms. Spike activity increased by $76.6 \%(2.8 \mathrm{AP})$ with PI increasing from 5 to $15 \mathrm{~ms}$ and it decreased by $57.2 \%$ (2.3 AP) when PI increased from 20 to $30 \mathrm{~ms}$. Toward longer PI, the relative activity of the neuron dropped further and was $<10 \%$. Thus, the PI-response function of B-LI4 was very similar to the tuning of phonotactic behavior, and both matched with a high correlation coefficient of $r^{2}=0.93$ and significance of $p<0.001$.

\section{Impact of $P P$}

The PP paradigm combines changes in PI and PD. The phonotactic response of females was strongest between PP-34 ms and PP-42 ms. The response pattern of local brain neurons to chirps with different $\mathrm{PP}$ are compared with the behavior in Figure 5.

B-LI2 responded to PP-18 ms with a continuous burst of spikes and generated $14.9 \pm 1.6 \mathrm{AP} /$ chirp; PP-34 ms evoked rhythmic activity coupled to the sound pulses with $15.3 \pm 4.9$ AP/chirp, and at PP-90 ms, each pulse elicited an EPSP with a burst of spikes and the response was $13.6 \pm 4.4 \mathrm{AP} /$ chirp (Fig. $5 \mathrm{~A}$, top). The PP-response function of B-LI2 showed that its relative spike activity remained always high and $>70 \%$ (Fig. $5 A$, bottom). Minor fluctuations in spike activity at different PP mirrored the differences in sound energy over the paradigm in a similar way as for TH1-AC1. The PP-response function of B-LI2 exhibited no optimum and did not correspond to the behavior as indicated by a correlation coefficient of $r^{2}=0.07(p>0.05)$. The PP-response function of B-LI2, however, matched the PP-response function of TH1-AC1 $\left(r^{2}=0.34 ; p<0.05\right)$.

In the contralaterally projecting neuron B-LC3, a PP-18 ms elicited a gradual slow depolarization with EPSPs coupled to the 
pattern of pulses and a mean response of $5.0 \pm 1.4 \mathrm{AP} /$ chirp (Fig. $5 B)$. At PP-34 ms, the mean spike activity increased to $15.3 \pm 2.5$ AP/chirp. The neuron responded only weakly to the first sound pulse of a chirp, strongest to pulses 2-4 with 3 AP and subsequently its activity decreased. Sound pulses at PP-90 ms elicited pronounced EPSPs of $10 \mathrm{mV}$ amplitude with a spike activity of $6.1 \pm 1.5 \mathrm{AP} /$ chirp. The PP-response function of B-LC3 revealed an optimum curve with a maximal response of $15.3 \mathrm{AP} /$ chirp at PP-34 ms. Spike activity increased by $66.3 \%$ when PP increased from 18 to $34 \mathrm{~ms}$ (10.4 AP/chirp) and decreased by $43.8 \%$ (6.9 AP) when PP increased further from 34 to $50 \mathrm{~ms}$. Thus the PP-response of B-LC3 was similar to phonotactic tuning at short PP but remained at higher amplitudes at long PP. Neural and phonotactic tuning matched highly significantly with $r^{2}=0.77(p<$ 0.001).

At PP-18 ms, neuron B-LI3 (Fig. 5C) did not respond until the fifth sound pulse and overall with $3.3 \pm 1.9 \mathrm{AP} /$ chirp. Its activity increased at PP-34 ms to $10.2 \pm$ 2.5 AP/chirp, and, like B-LC3, it generated its strongest response to pulses $2-4$ of the chirp. Activity decreased at PP-90 ms to $2.9 \pm 0.6 \mathrm{AP} /$ chirp; sound pulses elicited EPSPs with a spike response of 1-2 AP. The PP-response function of B-LI3 had the shape of an optimum curve with a maximum response at PP-34 ms. Spike activity increased by $64.3 \%$ (6.9 AP) with $\mathrm{PP}$ increasing from 18 to $34 \mathrm{~ms}$ and then decreased by $56.4 \%$ (6.1 AP) when PP increased from 34 to $58 \mathrm{~ms}$; it reached a minimal value of $28.3 \%$ at PP-98 ms. The PP-response function of B-LI3 matched the phonotactic tuning better than B-LC3 with a correlation coefficient of $r^{2}=0.92$ $(p<0.001)$.

The auditory response of B-LI4 was again characterized by inhibition and excitation and showed the best match to phonotactic behavior (Fig. 5D). At PP-18 ms, the first sound pulses of the chirps elicited an inhibition of $-4.3 \mathrm{mV}$, which was followed by a weak EPSP, generating $0.3 \pm 0.6 \mathrm{AP} /$ chirp. At PP-34 ms, the neuron generated $4.1 \pm 2.8 \mathrm{AP} /$ chirp. Its response started with an inhibition of $-3.4 \mathrm{mV}$ lasting for $\sim 40$ $\mathrm{ms}$. The consecutive sound pulses then elicited spikes that were followed by a mix of EPSPs and IPSPs toward the end of the response. Inhibition dominated the response to PP-90 ms. Sound pulses triggered an inhibition of -3 to $-5 \mathrm{mV}$ amplitude and 20-50 ms duration, and overall the neuron generated only $0.4 \pm$ 0.7 AP/chirp. The PP-response function of B-LI4 had an optimum curve with a maximum response at PP-34 ms. Spike activity increased by $95.2 \%$ (3.8 AP) with PP increasing from 18 to $34 \mathrm{~ms}$ and subsequently decreased by $85.5 \%$ (3.6 AP) when PP increased further from 34 to $58 \mathrm{~ms}$. Moreover, and different from B-LC3 and B-LI3, at very short or long PP, the response function had values $<5 \%$ and thereby had the highest match with phonotaxis, with a correlation coefficient of $r^{2}=0.94(p<0.001)$.

In comparison, there was no selectivity for species-specific pulse patterns at the level of the ascending interneuron TH1-AC1 and the brain neuron B-LI2. Selectivity for temporal patterns increased from B-LC3 and B-LI3 to B-LI4 (Table 2). B-LC3 and
Table 2. Correlation between phonotaxis and neural responses

\begin{tabular}{llll}
\hline Neurons & PD & PI & PP \\
\hline TH1-AC1 & $r^{2}=0.00(p>0.05)$ & $r^{2}=-0.18(p>0.05)$ & $r^{2}=0.00(p>0.05)$ \\
B-LI2 & $r^{2}=0.10(p>0.05)$ & $r^{2}=-0.25(p>0.05)$ & $r^{2}=0.07(p>0.05)$ \\
B-LC3 & $r^{2}=0.40(p=0.05)$ & $r^{2}=0.77(p<0.001)$ & $r^{2}=0.77(p<0.001)$ \\
B-LI3 & $r^{2}=0.46(p<0.05)$ & $r^{2}=0.75(p<0.001)$ & $r^{2}=0.92(p<0.001)$ \\
B-LL4 & $r^{2}=\mathbf{0 . 9 0}(p<\mathbf{0 . 0 0 1 )}$ & $r^{2}=\mathbf{0 . 9 3}(p<0.001)$ & $r^{2}=0.94(p<0.001)$
\end{tabular}

Correlation coefficient and significance between behavioral response data and neural response data given for all test patterns. Values in bold show a statistically significant match.
Behaviour

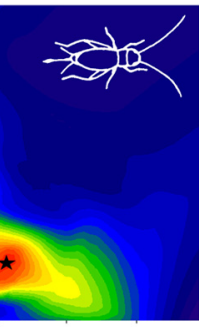

B-LC3
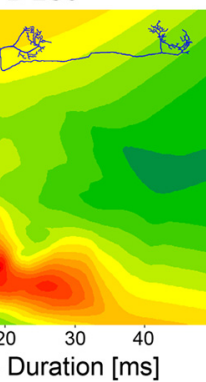

TH1-AC1

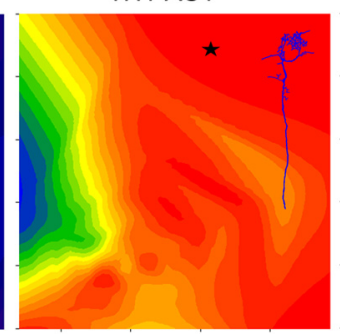

B-LI3

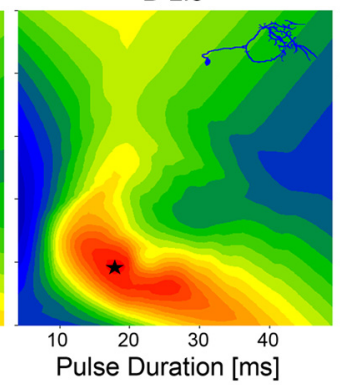

B-LI2

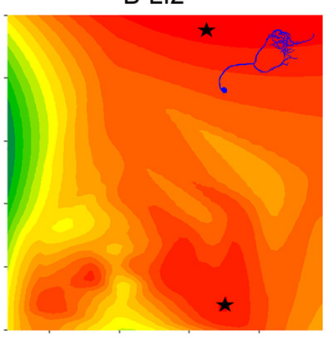

B-LI4

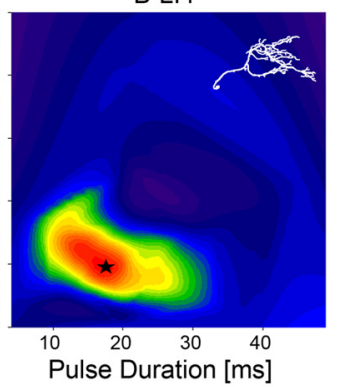

Colour code for relative responses of behaviour and neurons

$\square 0 \% \square 20 \% \square 40 \% \square 60 \% \square 80 \% \square 100 \%$

Figure 6. Relative responses of phonotaxis and brain neurons plotted against pulse duration (abscissa) and pulse interval (ordinate) based on all data points of the PD, PI, and PP test paradigms (e.g., data along the diagonal from the bottom left to the top right represent responses to the PP paradigm). In each plot, the $x$-axis starts at a pulse duration of $4 \mathrm{~ms}$. Responses are color coded with red showing the area of maximum response, indicated by asterisks.

B-LI3 revealed a significant match with behavior for different PI and PP, but only B-LI4 exhibited a highly significant match with the behavior over all test paradigms. The relative response of behavior and brain neurons was plotted in pulse duration/pulse interval profiles (Fig. 6). In the color coded diagrams, the areas of highest responses are indicated in red and marked by an asterisk. The diagrams demonstrate a close match between the tuning of the behavior and the response profile of B-LI4. TH1-AC1 and B-LI2 show a broad unspecific response profile, whereas B-LI3 and B-LC3 exhibit some degree of temporal tuning but are also activated over the whole range of pulse intervals tested.

Opposite to the improvement of pattern selectivity, the maximum spike activity in response to acoustic stimulation decreased by $\sim 90 \%$ from the ascending interneuron $\mathrm{TH} 1-\mathrm{AC} 1$ through to B-LI4 (Table 3). The average of the maximal responses over all test paradigms was $34.9 \mathrm{AP} /$ chirp for TH1-AC1, $14.3 \mathrm{AP} /$ chirp for B-LI2, 12.5 AP/chirp for B-LC3, 7.6 AP/chirp for B-LI3, and 3.9 AP/chirp for B-LI4. Whereas the overall spike activity decreased, the average spike latencies, as calculated for all sound pulses of chirps, increased from $20.7 \mathrm{~ms}$ (TH1-AC1) to $24.6 \mathrm{~ms}$ (B-LI2) to $31.6 \mathrm{~ms}$ (B-LC3) to $35.5 \mathrm{~ms}$ (B-LI3) and finally to 37.1 $\mathrm{ms}$ (B-LI4), indicating a sequenced flow of auditory activity in the pathway. In all five neurons tested, we found no evidence for 
Table 3. Average maximal spike activity of neurons

\begin{tabular}{lllll}
\hline Neurons & $P D$ & $\mathrm{PI}$ & $\mathrm{PP}$ & Average over all maxima (AP\chirp) \\
\hline TH1-AC1 & $40 \mathrm{~ms}$ & $100 \mathrm{~ms}$ & $74 \mathrm{~ms}$ & \\
& $37.3 \pm 5.1$ & $29.0 \pm 4.5$ & $38.4 \pm 5.0$ & $34.9 \pm 5.1$ \\
B-LI2 & $35 \mathrm{~ms}$ & $100 \mathrm{~ms}$ & $58 \mathrm{~ms}$ & \\
& $12.9 \pm 4.9$ & $14.4 \pm 1.3$ & $15.6 \pm 4.4$ & $14.3 \pm 1.3$ \\
B-LC3 & $20 \mathrm{~ms}$ & $15 \mathrm{~ms}$ & $34 \mathrm{~ms}$ & \\
& $12.0 \pm 1.1$ & $10.1 \pm 1.6$ & $15.3 \pm 2.5$ & $12.5 \pm 2.6$ \\
B-LI3 & $20 \mathrm{~ms}$ & $15 \mathrm{~ms}$ & $34 \mathrm{~ms}$ & \\
& $6.7 \pm 1.1$ & $5.9 \pm 0.9$ & $10.2 \pm 2.5$ & $7.6 \pm 2.3$ \\
B-LI4 & $15 \mathrm{~ms}$ & $20 \mathrm{~ms}$ & $34 \mathrm{~ms}$ & \\
& $4.7 \pm 0.2$ & $3 \pm 1.5$ & $4.1 \pm 2.8$ & $3.9 \pm 0.9$ \\
\hline
\end{tabular}

Spike activity of the neurons (AP\chirp $\pm S D$ ) at their maximal response given for all test paradigms. Average activity (AP\chirp) over all response maxima of all test paradigms is given in the right column.

ongoing maintained oscillations of the membrane potential after stimulation with a calling-song-like sound pattern.

The response of selective neurons was higher to PD-20 ms compared with PI-20 ms, although the corresponding chirps were identical. In B-LI4, a coincidental difference of only one spike led to a relative difference of $30 \%$. Habituation in the auditory pathway may have contributed to this difference. In the pulse duration paradigm, the chirps preceding the test with $20 \mathrm{~ms}$ pulse duration had an overall lower sound energy as the chirps preceding the corresponding test in the pulse interval paradigm. Correspondingly, the PI response function showed left to its maximum a higher spike activity than the PD response function.

\section{Temporal dynamic underlying pattern selectivity}

Temporal pattern recognition requires at least two consecutive sound pulses to allow an analysis of the pulse rate (Poulet and Hedwig, 2005). How many pulses and intervals does the system require for a selective response to specific PI? By using a test paradigm with a successive variation of the pulse interval, we tested whether temporal filtering is established on a pulse-topulse basis after a specific pulse interval, or whether pattern recognition requires integrating over several sound pulses and intervals. We tested the response of TH1-AC1 and B-LC3 by systematically altering the pulse intervals in a sequence of six pulses. The PI between the first and second pulse had a duration of $21 \mathrm{~ms}$ based on the species-specific pattern. It was followed by pulses with a preceding PI of 31, 41, 71, and again $21 \mathrm{~ms}$. This sequence was repeated after $230 \mathrm{~ms}$. The resulting PST histograms and the average of the instantaneous spike frequency were calculated over 40 stimuli for TH1-AC1 (Fig. 7A) and over 10 stimuli for B-LC3 in a female (Fig. $7 B$ ), which showed an exceptional selectivity for specific PI. In the following, we provide the mean data for TH1-AC1 and B-LC3 obtained in three females, respectively; each neuron was tested with 10 sequences.

TH1-AC1 responded to each sound pulse of the paradigm with a mean of $7.1 \pm 0.5$ spikes (Fig. $7 A$ ). Over the whole sequence, its response to the consecutive pulses gradually dropped from $7.9 \pm 1.4$ to $6.6 \pm 0.7 \mathrm{AP}$. The mean spike frequency of TH1-AC1 was in the range of $346-384 \mathrm{~Hz}$ for all pulses (Fig. 7A, bottom). As with the previous paradigms, interneuron TH1-AC1 exhibited no selectivity for any PI (Fig. 7B); for example, the activity of TH1-AC1 even dropped from $7.9 \pm 1.4$ AP after a PI of $230 \mathrm{~ms}$ to $7.3 \pm 0.9 \mathrm{AP}$ in response to the sound pulse after a PI of $21 \mathrm{~ms}$.

In contrast, the activity of B-LC3 did depend on the duration of the interval preceding a sound pulse (Fig. $7 B$ ). The first sound pulse of the sequence occurred after a PI of $230 \mathrm{~ms}$ and elicited $1.9 \pm 0.4 \mathrm{AP}$ and a mean spike rate of $84.79 \mathrm{~Hz}$. The second pulse, occurring after an interval of $21 \mathrm{~ms}$, elicited a considerably stronger response with $3 \pm 0.5 \mathrm{AP} /$ sound pulse and a spike rate of $202.6 \mathrm{~Hz}$. The response to the third pulse after an interval of 31 ms was only $2.1 \pm 0.8 \mathrm{AP}$ with a spike rate $127.2 \mathrm{~Hz}$. After the following even longer intervals of 41 and $71 \mathrm{~ms}$, the neuron responded only with $1.2 \pm 0.8 \mathrm{AP} /$ sound pulse ( $47.3 \mathrm{~Hz}$ spike rate) and $1.5 \pm 0.8 \mathrm{AP} /$ sound pulse $(78.4 \mathrm{~Hz}$ spike rate), respectively. However, to the last pulse of the sequence, which again was presented after an interval of $21 \mathrm{~ms}$, the neuron generated a strong response with $2.4 \pm 0.6 \mathrm{AP}$ with a spike rate of $178.9 \mathrm{~Hz}$. In the PST histogram and the average of the instantaneous spike rate of a selected female (Fig. $7 B$, bottom), the enhanced responses of B-LC3 to sound pulses occurring after an interval of $21 \mathrm{~ms}$ clearly stand out. This test paradigm therefore indicates that temporal filtering as reflected by the B-LC3 neuron is based on a pulse-topulse processing and that the response to a pulse crucially depends on the duration of the preceding pulse interval. The response was particular strong, when the preceding interval matched the species-specific pulse interval.

For interneuron B-LI4, time interval-dependent changes in synaptic and spike activity could be tested more systematically with pairs of pulses for which PI was varied in 5-10 ms increments (Fig. 7C). A single sound pulse elicited an IPSP with a duration of $\sim 17 \mathrm{~ms}$ that was followed by a subthreshold EPSP after which the response returned to the resting potential. After that, there was no indication of an oscillatory response of the B-LI4 membrane. At a PI of $5 \mathrm{~ms}$, the EPSP was followed by a pronounced inhibition lasting for $45 \mathrm{~ms}$ (Fig. 7, asterisk). At PI-10 ms, however, the response to the second pulse started with an inhibition, but then elicited a small EPSP lasting $15 \mathrm{~ms}$ (Fig. 7, arrowhead). In the following tests with PI increasing from 15 to $40 \mathrm{~ms}$, the excitatory response to the second pulse considerably increased as B-LI4 generated suprathreshold EPSPs with 1-2 AP. There was no indication of membrane potential oscillation even after the strongest response to the second pulse at a PI of $25 \mathrm{~ms}$ $(1.2 \pm 0.5 \mathrm{AP} /$ pulse $)$. At a PI of $50 \mathrm{~ms}$, the second sound pulse only caused a weak EPSP. At PI-60 ms, B-LI4 responded to the second sound pulse again with an inhibition and a consecutive subthreshold EPSP, in the same way as it responded to the first pulse of the stimulus pair. In the recordings, we obtained a strong excitatory response to the second pulse at PI-15 to $40 \mathrm{~ms}$. When tested with different PI, the neurons PI-response function was maximal at $20 \mathrm{~ms}$ (Fig. 4D). Temporal selectivity as measured over a whole chirp may, however, be sharpened when several sound pulses with the according PI are presented. This test clearly demonstrated that the selective response properties of interneuron B-LI4 for specific PI depend on the time interval between two consecutive pulses due to an interaction of inhibitory and excitatory PSPs.

\section{Discussion}

\section{Temporal tuning of cricket phonotaxis}

Phonotaxis in female crickets ( $G$. bimaculatus, G. campestris) is tuned to the temporal features of the male calling song and shows bandpass characteristics when tested with different pulse periods (Popov and Shuvalov, 1977; Thorson et al., 1982; Doherty, 1985a; Hedwig, 2006). The behavior is additionally affected by variation in pulse interval, pulse duration, and also pulse number and chirp interval (Doherty, 1985a; Stout and McGhee, 1988; Hennig, 2009). With a trackball system (Hedwig and Poulet, 2004), we recorded the characteristic tuning of female phonotaxis to variations of pulse patterns. Phonotaxis was strongest at $15 \mathrm{~ms}$ pulse duration, $15 \mathrm{~ms}$ pulse interval, and $34-42 \mathrm{~ms}$ pulse period. These 
parameters fall well within the range of the male calling song parameters (Doherty, 1985b; Ferreira and Ferguson, 2002; Verburgt et al., 2010).

\section{Structure of local auditory neurons within the brain}

The prothoracic ascending neuron TH1$\mathrm{AC} 1$ is the only neuron that forwards calling-song-specific auditory information to the brain (Wohlers and Huber, 1982; Schildberger, 1984). In the anterior protocerebrum, its two main axonal projections run anteriorly and posteriorly around an unidentified structure and form a dense ring-like arborization. We identified local brain neurons (B-LI2, B-LI3, and B-LI4) with a lateral cell body and a similar ring-like pattern of arborizations that overlap the projections of TH1AC1. These neurons showed no clear separation of dendritic and axonal branches, which is typical for local sensory interneurons (Burrows, 1992). Structural characteristics alone made distinguishing between B-LI3 and B-LI4 difficult, but they revealed very different physiological properties, particularly in the degree of inhibition resulting from sound pulses. B-LC3 connected both auditory areas at the anterior protocerebrum. Its ipsilateral branches exhibited a smooth dendritic appearance, and its contralateral projections, a beaded axonal structure (Peters et al., 1986). Bilateral auditory neuropils are also linked in the brain of bushcrickets (Ostrowski, 2009) and locusts (Boyan et al., 1993), a feature that may support pattern recognition and directional processing.

Other identified auditory brain neurons also showed dense branching patterns overlapping with the ring-like arborization area (Schildberger, 1984, 1985). Therefore, we consider that this area is a crucial early stage for auditory processing in the cricket brain.

\section{Temporal selectivity: brain neurons and phonotactic behavior}

When we compared the tuning of phonotactic behavior with that of auditory neurons, the response functions of TH1-AC1 revealed no temporal selectivity (Fig. 2); its spike activity forwarded any pattern of acoustic stimuli to the brain. Local brain neuron B-LI2 also showed no selectivity (Figs. 3-5), and as its response functions were very similar to $\mathrm{TH} 1-\mathrm{AC} 1$, its activity might be directly driven by the ascending interneuron.

Three auditory brain neurons (B-LC3, B-LI3, and B-LI4) revealed a clear temporal selectivity that matched the tuning of phonotaxis to different degrees (Figs. 3-5). The responses of B-LI3 and B-LC3 were quite similar. When tested with a chirp pattern akin to calling song, both neurons had a stronger response to the second pulse of the chirp, showing that they received a more complex input than just a reflection of the ascending spike pattern. When $>4$ pulses/chirp were presented to B-LI3 and B-LC3 (Fig. 5B, C), the response toward the sound pulses over a chirp decreased to a stronger degree than expected based on the ascending activity (Fig. 2C). Although these results indicate the involvement of inhibitory processing, IPSPs were not obvious in these interneurons. The response optima and the shape of the neural response functions matched the behavior; however, away from the optimum, the minimum response was often $>50 \%$.

Response functions were quite different for neuron B-LI4, which demonstrated the highest temporal selectivity and best match to phonotaxis (Figs. 3-6). B-LI4 generated pronounced IPSPs at the beginning of each response to a chirp. The inhibitory inputs dominated when a chirp was clearly different from the calling song pattern, reducing its spike activity to very low levels (Figs. 3D-5D). Thus, the maximum responses were always well above background activity, and all of the response functions of B-LI4 exhibited a close match to the tuning of phonotaxis. This revealed that bandpass-like selectivity for auditory pulse patterns as exhibited by phonotactic behavior is already established within the ring-like arborization area in the anterior protocerebrum. Every brain neuron we analyzed exhibited a clear phasic response to individual sound pulses when exposed to a calling-song-like pattern. In relation to the level of temporal filtering, however, the number of AP/chirp continuously decreased from TH1-AC1 to $\mathrm{B}-\mathrm{LI} 2$ to B-LC3 to B-LI3 to reach nearly a $90 \%$ reduction in B-LI4. This decrease of spike activity is in line with concepts on sparse coding (Olshausen and Field, 2004). It shifts the representation of stimulus features from a temporal activity-based code to a neuron-specific place code and appears to be an energetically efficient way for simple networks to ensure a robust representation of stimulus patterns.

\section{Neural mechanisms underlying temporal selectivity}

Different concepts have been proposed to explain the selectivity of cricket phonotaxis. Hoy (1978), Weber and Thorson (1989), and Hennig (2003) suggested that temporal filtering might depend on "feature detection" when the temporal pattern of the signal coincides with a form of template matching or crosscorrelation. As B-LI4 revealed a strong selectivity in its response, it may be described as a "feature detector" for the calling song, considering, however, that inhibitory and excitatory inputs from the presynaptic auditory network shape its activity. As the neuron showed a tuning to $\mathrm{PD}$, even when the pulse period was kept constant, its tuning does not appear to depend on the pulse rate but rather on combinations of PD and PI. Furthermore, a pulse 
coincidence detector was suggested, based on autocorrelation via a delay line adjusted to the species-specific pulse interval (Reiss, 1964; Weber and Thorson, 1989). Anatomical substrates for 40 ms delay lines are unlikely in insect brains; however, delays may be achieved by inhibitory processing as evident in B-LI4.

Evidence from Bush and Schul (2006) indicated that pattern selectivity may result from intrinsic membrane potential oscillations of auditory neurons resonating with the frequency of the species-specific pulse pattern. Our recordings did not provide evidence for resonance in the auditory network as stimulation with calling song patterns did not lead to ongoing oscillations of the membrane potential of any of the neurons recorded. Furthermore, neither our behavioral data nor the response functions of our neurons revealed a secondary peak at one-half of the optimal pulse period as suggested by Bush and Schul (2006). Only the membrane potential of B-LC3 in some preparations transiently oscillated at $\sim 34 \mathrm{~Hz}$ when stimulated with a constant tone of 160 ms (Fig. 3B, top), maybe reflecting feedback loops of the network tuned to the temporal features of the calling song.

Schildberger (1984) suggested that temporal selectivity underlying phonotaxis results from low-pass and high-pass filter neurons that together shape a bandpass response in other brain neurons, a concept akin to bandpass responses encountered in the anuran auditory pathway (Rose and Capranica, 1983). Bandpass responses via low- and high-pass filtering require at least two sound pulses and long latency processing. Phonotactic orientation, however, works much faster and can occur toward individual sound pulses (Hedwig and Poulet, 2004, 2005). Here, we show that selective processing in brain neurons requires only one specific pulse interval to elicit an enhanced response to the consecutive sound pulse (Fig. 7); a response property that indicates a change in auditory responsiveness over time, which is very different from bandpass filtering by low-pass and high-pass neurons.

Thus, pattern recognition seems to be tightly linked to specific changes in neural responsiveness occurring over the duration of a single pulse interval. Considering the synaptic activity of B-LI4 (Fig. 7C), which exhibited clear bandpass properties (Figs. 3D$5 D$ ), we propose that species-specific pattern recognition in the brain of $G$. bimaculatus is based on fast pulse-by-pulse interactions of inhibitory and excitatory synaptic activity in local brain neurons. Neuron B-LI4 revealed phasic responses to individual sound pulses and may only be a few synapses after TH1-AC1, whereas the bandpass neuron described by Schildberger (1984) generated a rather tonic pattern of spikes over a chirp and did not overlap with the ring-like arborizations, indicating that those bandpass responses resulted from preceding auditory filtering.

Concepts for temporal processing in lower vertebrates highlight the importance of inhibitory inputs for interval selectivity and of delays established by inhibition lasting longer than the duration of single sound pulses. A model by Large and Crawford (2002) demonstrated that temporal selectivity in the fish Pollimyrus could be facilitated by the timing of excitatory inputs that coincide with an intrinsic postinhibitory rebound excitation. The importance of the interaction between inhibition and pulse ratedependent excitation for interval selectivity has been shown in anurans (Edwards et al., 2007; Rose et al., 2011). "Intervalcounting" neurons responded with spikes only after some pulse intervals with a specific pulse repetition rate due to a gradual decrease of the inhibitory input and thus increasing excitation. Like in the cricket, these responses in lower vertebrates support a model based on integration of EPSPs and IPSPs in single neurons (Buonomano, 2000) to which postinhibitory rebound mechanisms may contribute.

\section{References}

Boyan G, Williams L, Meier T (1993) Organization of the commissural fibers in the adult brain of the locust. J Comp Neurol 332:358-377.

Bradbury JW, Vehrenkamp SL (1998) Principles of animal communication. Sunderland, MA: Sinauer Associates.

Brodfuehrer PD, Hoy RR (1990) Ultrasound sensitive neurons in the cricket brain. J Comp Physiol A Neuroethol Sens Neural Behav Physiol 166:651-662.

Buonomano DV (2000) Decoding temporal information: a model based on short-term synaptic plasticity. J Neurosci 20:1129-1141.

Burrows M (1992) Local circuits for the control of leg movements in an insect. Trends Neurosci 15:226-232.

Bush SL, Schul J (2006) Pulse-rate recognition in an insect: evidence of a role for oscillatory neurons. J Comp Physiol A Neuroethol Sens Neural Behav Physiol 192:113-121.

Crawford JD (1997) Feature-detecting auditory neurons in the brain of a sound-producing fish. J Comp Physiol A Neuroethol Sens Neural Behav Physiol 180:439-450.

Doherty JA (1985a) Trade-off phenomena in calling song recognition and phonotaxis in the cricket Gryllus bimaculatus (Orthoptera, Gryllidae). J Comp Physiol A Neuroethol Sens Neural Behav Physiol 156:787-801.

Doherty JA (1985b) Temperature coupling and "trade off" phenomena in the acoustic communication system of the cricket, Gryllus bimaculatus De Geer. J Exp Biol 114:17-35.

Edwards CJ, Leary CJ, Rose GJ (2007) Counting on inhibition and ratedependent excitation in the auditory system. J Neurosci 27:13384-13392.

Endler JA, Basolo AL (1998) Sensory ecology, receiver biases and sexual selection. Tree 13:415-420.

Fay RR (1992) Structure and function in sound discrimination among vertebrates. In: The evolutionary biology of hearing (Webster D, Fay RR, Popper AN, eds), pp 229-263. Springer, New York.

Ferreira M, Ferguson JWH (2002) Geographic variation in the calling song of the field cricket Gryllus bimaculatus (Orthoptera: Gryllidae) and its relevance to mate recognition and mate choice. J Zool Lond 257:163-170.

Gerhardt HC, Huber F (2002) Acoustic communication in insects and anurans. Chicago: The University of Chicago.

Hedwig B (1986) On the role in stridulation of plurisegmental interneurons of the acridid grasshopper Omocestus viridulus L. I. Anatomy and physiology of descending cephalothoracic interneurons. J Comp Physiol A Neuroethol Sens Neural Behav Physiol 158:413-427.

Hedwig B (2006) Pulses, patterns and paths: neurobiology of acoustic behavior in crickets. J Comp Physiol A Neuroethol Sens Neural Behav Physiol 192:677-689.

Hedwig B, Knepper M (1992) NEUROLAB, a comprehensive program for the analysis of neurophysiological and behavioral data. J Neurosci Methods 45:135-148.

Hedwig B, Poulet JF (2004) Complex auditory behavior emerges from simple reactive steering. Nature 430:781-785.

Hedwig B, Poulet JF (2005) Mechanisms underlying phonotactic steering in the cricket Gryllus bimaculatus revealed with a fast trackball system. J Exp Biol 208:915-927.

Hennig RM (2003) Acoustic feature extraction by cross-correlation in crickets? J Comp Physiol A Neuroethol Sens Neural Behav Physiol 189:589-598

Hennig RM (2009) Walking in Fourier's space: algorithms for the computation of periodicities in song patterns by the cricket Gryllus bimaculatus. J Comp Physiol A Neuroethol Sens Neural Behav Physiol 195:971-987.

Hoy RR (1978) Acoustic communication in crickets: a model system for the study of feature detection. Fed Proc 37:2316-2323.

Knepper M, Hedwig B (1997) NEUROLAB, a PC-program for the processing of neurobiological data. Comput Methods Programs Biomed 52:75-77.

Large EW, Crawford JD (2002) Auditory temporal computation: interval selectivity based on post-inhibitory rebound. J Comput Neurosci 13:125-142.

Nabatiyan A, Poulet JF, de Polavieja GG, Hedwig B (2003) Temporal pattern recognition based on instantaneous spike rate coding in a simple auditory system. J Neurophysiol 90:2484-2493.

Olshausen BA, Field DJ (2004) Sparse coding of sensory inputs. Curr Opin Neurobiol 14:481-487.

Ostrowski TD (2009) Filtering of species-specific song parameters via in- 
terneurons in a bush cricket's brain. Dissertation, Universitaet Goettingen, Goettingen, Germany.

Peters BH, Römer H, Marquart V (1986) Spatial segregation of synaptic inputs and outputs in a locust auditory interneurone. J Comp Neurol 254:34-50.

Pollack G (2000) Who, what, where? Recognition and localization of acoustic signals by insects. Curr Opin Neurobiol 10:763-767.

Pollack GS, Hoy RR (1979) Temporal pattern as a cue for species-specific calling song recognition in crickets. Science 204:429-432.

Popov AV, Shuvalov VF (1977) Phonotactic behavior of crickets. J Comp Physiol A Neuroethol Sens Neural Behav Physiol 119:111-126.

Poulet JF, Hedwig B (2005) Auditory orientation in crickets: pattern recognition controls reactive steering. Proc Natl Acad Sci U S A 102:15665-15669.

Reiss RF (1964) A theory of resonance. In: Neural theory and modelling (Reiss RF, ed), pp 105-137. Stanford, CA: Stanford UP.

Rose G, Capranica RR (1983) Temporal selectivity in the central auditory system of the Leopard frog. Science 219:1087-1089.

Rose GJ, Leary CJ, Edwards CJ (2011) Interval-counting neurons in the anuran auditory midbrain: factors underlying diversity of interval tuning. J Comp Physiol A Neuroethol Sens Neural Behav Physiol 197:97-108.

Ryan MJ (1998) Sexual selection, receiver biases, and the evolution of sex differences. Science 281:1999-2003.

Schildberger K (1984) Temporal selectivity of identified auditory neurons in the cricket brain. J Comp Physiol A Neuroethol Sens Neural Behav Physiol 155:171-185.

Schildberger K (1985) Recognition of temporal patterns by identified auditory neurons in the cricket brain. In: Acoustic and vibrational communication in insects (Kalmring K, Eisner N, eds), pp 41-49. Hamburg, Germany: Paul-Parey Verlag.
Schildberger K, Hoerner M (1988) The function of auditory neurons in cricket phonotaxis: I. Influence of hyperpolarization of identified neurons on sound localization. J Comp Physiol A Neuroethol Sens Neural Behav Physiol 163:621-631.

Stout J, McGhee R (1988) Attractiveness of the male Acheta domesticus calling song to females. II. The relative importance of syllable period, intensity and chirp rate. J Comp Physiol A Neuroethol Sens Neural Behav Physiol 164:277-287.

Thorson J, Weber T, Huber F (1982) Auditory behaviour of the cricket. II. Simplicity of calling-song recognition in Gryllus, and anomalous phonotaxis at abnormal carrier frequencies. J Comp Physiol A Neuroethol Sens Neural Behav Physiol 146:361-378.

Verburgt L, Ferguson JW, Weber T (2008) Phonotactic response of female crickets on the Kramer treadmill: methodology, sensory and behavioural implications. J Comp Physiol A Neuroethol Sens Neural Behav Physiol 194:79-96.

Verburgt L, Ferreira M, Ferguson JWH (2010) Male field cricket song reflects age, allowing females to prefer young males. Anim Behav 81:19-29.

Weber T, Thorson J (1989) Phonotactic behavior of walking crickets. In: Cricket behaviour and neurobiology (Huber F, Moore TE, Loher W, eds), pp 310-339. Ithaca, NY: Cornell UP.

Wohlers DW, Huber F (1982) Processing of sound signals by six types of neurons in the prothoracic ganglion of the cricket, Gryllus campestris L. J Comp Physiol A Neuroethol Sens Neural Behav Physiol 146:161-173.

Yu AC, Margoliash D (1996) Temporal hierarchical control of singing in birds. Science 273:1871-1875.

Zorović M, Hedwig B (2011) Processing of species-specific auditory patterns in the cricket brain by ascending, local and descending neurons during standing and walking. J Neurophysiol 105:2181-2194. 